\title{
Implementación de métodos de procesamiento de señales EEG para aplicaciones de comunicación y control
}

\section{Implementation of EEG signal processing methods for communication and control application}

\author{
Shirley Cordova Villar ${ }^{1}$, Willian A. Perez Oviedo ${ }^{1}$, Avid Román Gonzalez ${ }^{12}$ \\ ${ }^{1}$ Universidad Nacional San Antonio Abad del Cusco \\ 2 TELECOM ParisTech, 46 rue Barrault, 75013 - Paris, Francia
}

DOI: https://doi.org/10.33017/RevECIPeru2013.0004/

\section{Resumen}

La interface cerebro-computador (ICC) es un instrumento de comunicación entre la mente o la función cognitiva del ser humano y el ambiente externo, esta función mental es creada por el cerebro; las señales son capturadas, pre-procesadas y puestas en un clasificador. Este artículo tiene como objetivo la implementación y comparación de algoritmos basados en diferentes métodos de procesamiento de señales EEG para aplicaciones ICC que actualmente existen, para encontrar que método proporciona mejores resultados.

Descriptores: ICC, EEG, procesamiento de señales, parámetros AAR, clasificación, comunicación y control, pensamiento

\section{Abstract}

The Brain Computer Interface $(\mathrm{BCl})$ is a communication instrument between mental or cognitive human function and the external environment, this mental function is created by the brain; the signals are captured, pre-processed and put into a classifier. This article aims to implement and compare the algorithms based on different methods of EEG signal processing for $\mathrm{BCl}$ applications that currently exist, in order to find methods whose algorithms provide better results.

Keywords: BCl, EEG, signal processing, AAR parameters, classification, communication and control, thought

\section{Introducción}

A partir de que se ha desarrollado la tecnología que permite capturar las señales electroencefalograficas (EEG), se ha venido investigando la posibilidad de interpretarlas y usarlas como un medio de comunicación o control de dispositivos. Este tipo de tecnología es conocido como $\mathrm{BCl}$ por sus siglas en inglés (Brain - Computer Interface). Con los diferentes trabajos previos de investigación acerca del tema como [1], [2], [3], se descubren y presentan cada vez nuevas dificultades en la forma de cómo hacer que los datos tomados a partir de un electroencefalograma sean procesados de tal forma que los resultados sean lo suficientemente confiables, por lo que es necesario la implementación de métodos que permitan el desarrollo de este tipo de interfaces de manera eficiente y confiable.

En la actualidad se cuenta con varias alternativas para la implementación de BCls, pero no se sabe a ciencia cierta cuál es el método más efectivo para hacer confiables estos dispositivos, por lo que el presente trabajo está orientado a analizar los diferentes métodos de procesamiento de señales EEG para aplicaciones $\mathrm{BCl}$ existentes con el objetivo de encontrar el algoritmo óptimo para el desarrollo de aplicaciones de $\mathrm{BCl}$. Las $\mathrm{BCl}$ son utilizadas principalmente para personas discapacitadas por diferentes motivos descritos en [3], [4], [5], [6], [7]. 
Es necesario mencionar que la metodología usada para el análisis de las señales EEG desarrolladas hasta la fecha, no garantizan una máxima eficiencia para los diversos métodos de diseño de una interfaz Cerebro-Computadora, ya que como se verá más adelante existen diversos puntos de partida y por consiguiente métodos de estudio e implementación de cada etapa de una $\mathrm{BCl}$.

Dentro de esta área de investigación existen muchos puntos que aún hay que estudiar para así resolver algunos problemas que aún siguen vigentes, como lo es la optimización del tiempo de entrenamiento y la precisión para interpretar los comandos del usuario. No obstante, este trabajo centrará su investigación dentro de puntos específicos y primordiales, los cuales son:

- La caracterización de las señales EEG (extracción de características.

- La clasificación de las señales EEG.

\section{Marco Teórico}

\section{Señales EEG}

La Electroencefalografía es el registro y evaluación de los potenciales eléctricos generados por el cerebro y obtenidos por medio de electrodos situados sobre la superficie del cuero cabelludo. El electroencefalograma (EEG) es el registro de la actividad eléctrica de las neuronas del encéfalo. Dicho registro posee formas muy complejas que varían mucho con la localización de los electrodos y entre individuos. Esto es debido al gran número de interconexiones que presentan las neuronas y por la estructura no uniforme del encéfalo.

Las señales EEG tiene diferentes ritmos dentro de la banda de frecuencia con las siguientes características: [1] [8]

Ritmo Alfa o Mu: Voltaje bajo (20-60 $\mu \mathrm{v} / 3-4 \mathrm{~mm})$ con morfología variable. Frecuencia alta $(8-13 \mathrm{~Hz})$.

Ritmo Beta: Voltaje bajo (10-15 $\mu \mathrm{v} /$ 1-1.5 mm) con morfología variable. Frecuencia alta (13-25 ó + Hz).

Ritmo Tetha: Voltaje alto (50 $\mu \mathrm{v} / 7 \mathrm{~mm})$. Frecuencia $(4-8 \mathrm{~Hz})$.

Ritmo Delta: Voltaje alto (70 -100 $\mu \mathrm{v} / \mathrm{g}-14 \mathrm{~mm})$ con morfología variable. Frecuencia baja (4 ó - Hz).

Sistema internacional de posicionamiento de electrodos 10/20
El sistema internacional 10/20 es el más utilizado en el momento actual. Para situar los electrodos según este sistema se procede de la forma siguiente: El electrodo inactivo o común se coloca alejado del cráneo (lóbulo de la oreja, nariz o mentón). Se cuenta con puntos de referencia tales como: nasion e inion. Diez por ciento por encima de los puntos de referencia se encuentran los planos pre frontal y occipital. El resto está dividido en cuatro partes iguales de $20 \%$ cada una.
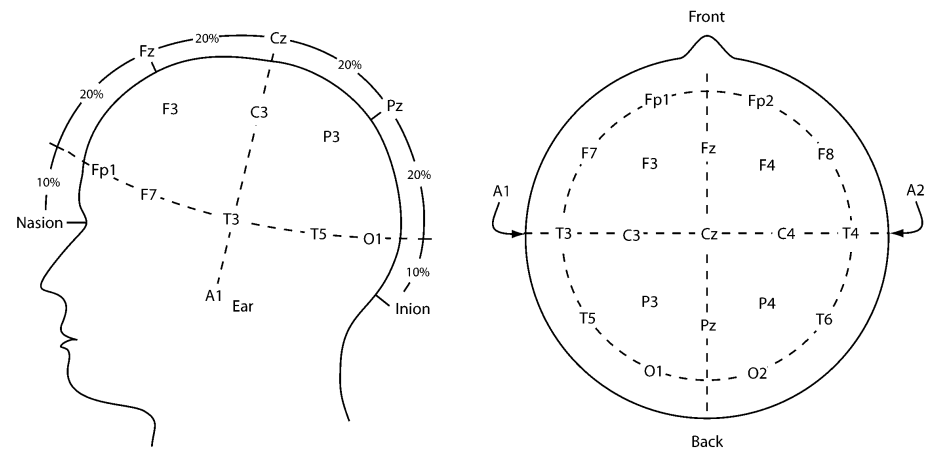

Figura 1: Posicionamiento de los Electrodos

\section{Las Interfaces Cerebro-Computador}

El concepto de una Interfaz Cerebro-Computador (BCl) parece relativamente sencillo: se mide la actividad del cerebro, se interpreta esta actividad de forma que determinados diferentes patrones de pensamiento que puedan ser detectados $y$, por último, se comunica con el sistema externo que se desea controlar o con quien se desea comunicar. El diagrama de bloques general para un $\mathrm{BCl}$ se muestra en la Figura 2.

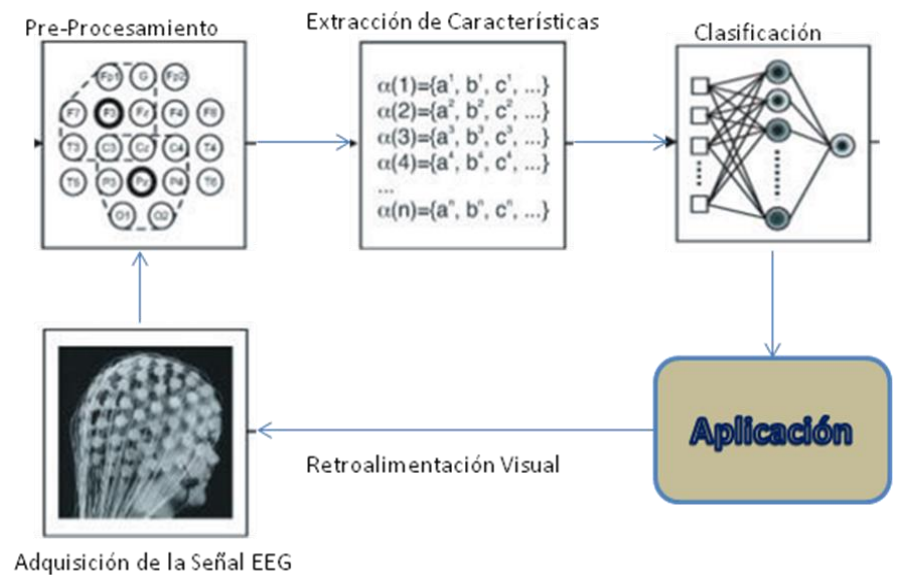

Figura 2: Diagrama de bloques general para un $\mathrm{BCl}$ [2]

La etapa de extracción de características es probablemente el paso más crítico en el procesado de la señal EEG, ya que determina directamente 
tanto el rendimiento como la precisión del algoritmo de clasificación utilizado. Distintos criterios pueden ser observados a la hora de llevar a cabo el proceso de extracción, a partir de las señales EEG medidas, de un conjunto de características válido para la clasificación de los estados mentales considerados Para la extracción de características existen muchos métodos como: Los parámetros autoregresivos utilizados en [1] [13], el promedio de la transformada rápida de Fourier (FFT) utilizado en [9], el promedio de la señal en el dominio del tiempo por ventanas utilizado en [10], análisis de componentes independientes en [11] [12], etc.

La fase de clasificación es la tarea final del procesado. La entrada al algoritmo de clasificación es el conjunto de características extraídas en la etapa anterior y la salida es una indicación del estado mental del usuario. En este caso estamos trabajando con dos estados: izquierda y derecha. Al igual que en el paso anterior, existen varios métodos para clasificar los datos, los cuales son explicados en cada uno de los artículos mencionados en las referencias, en especial en [14].

\section{Métodos de Extracción de Características}

El ritmo mu, que corresponde a una oscilación de la señal EEG comprendida entre los 8 y $13 \mathrm{~Hz}$, es captado en la zona sensomotora situada en la región central del cuero cabelludo. Dicho ritmo, presente en la mayoría de los adultos, tiene la particularidad de presentar una atenuación en su amplitud cuando se lleva a cabo algún tipo de movimiento, o lo que es más importante cuando se tiene la intención de realizar algún movimiento, 0 sencillamente imaginando movimientos de las extremidades, tal y como se muestra en las figuras 3 y 4 . Es justamente este comportamiento que debemos caracterizar mediante los métodos descritos en adelante.

Extracción de características para toda la señal mediante Transformada Rápida de Fourier

La Transformada de Fourier nos permite ver los componentes de frecuencia (y amplitud) que tiene una señal EEG en forma gráfica. Se utiliza la FFT (Fast Fourier Transform) para tomar muestras frecuenciales de la señal a procesar.

Al observar la gráfica del espectro de las señales EEG, en la figura 6 se observa un pico máximo en la banda mu, por lo que se toma este valor como una característica de la señal EEG, como segundo valor característico se toma el valor promedio de la señal lo que hacen un total de 4 características para cada prueba (2 para cada electrodo).

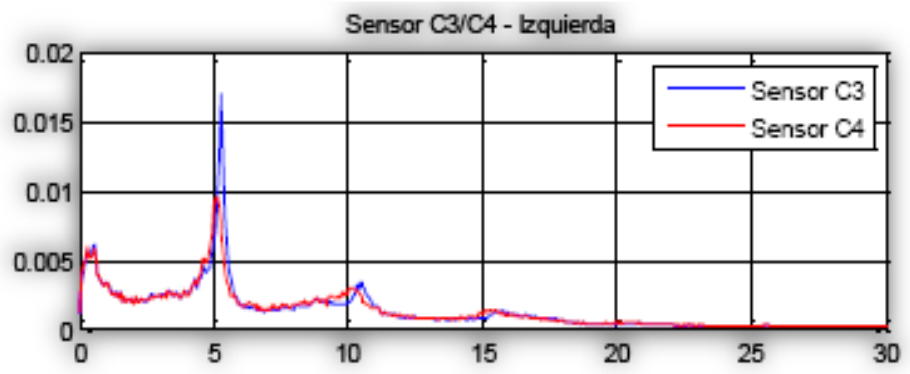

Sensor C3/C4 - Derecha

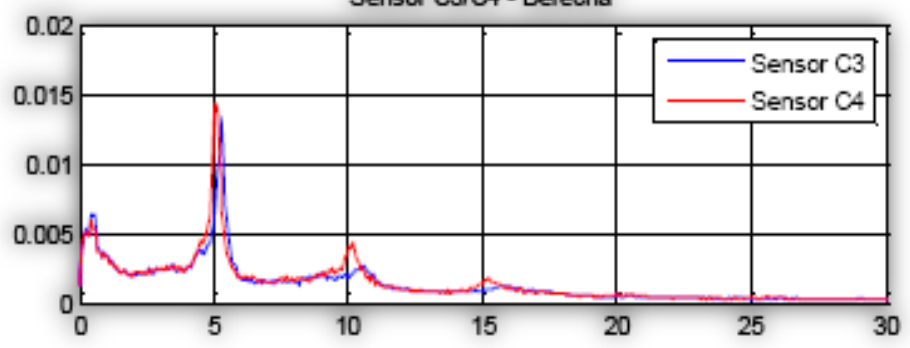

Figura 3: Señales promedio de las pruebas elaboradas. Cuando se piensa en Izquierda, el sensor C4 ubicado en el lóbulo derecho experimenta una disminución de amplitud de la señal. Cuando se piensa en Derecha, el sensor C3 ubicado en el lóbulo izquierdo experimenta una disminución de amplitud de la señal.

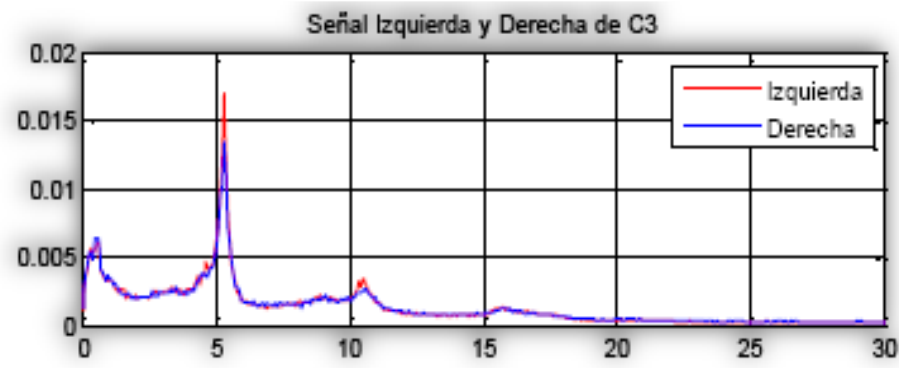

Señal Izquierda y Derecha de C4

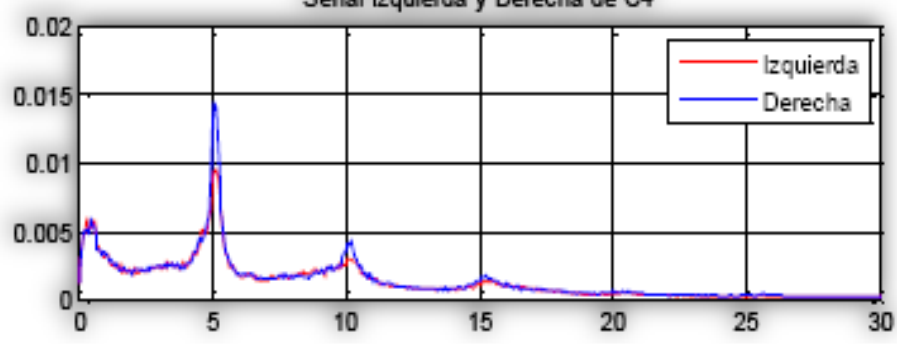

Figura 4: Señales promedio de las pruebas elaboradas por Sensor. El sensor C3 muestra señales derechas con menor amplitud en comparación con la izquierda debido a que es el área que controla el lado derechos del cuerpo. El sensor C4 muestra señales izquierdas con menor amplitud en comparación con la derecha debido a que es el área que controla el lado izquierdo del cuerpo. 
Figura 6: Espectro de frecuencias de la Señal EEG

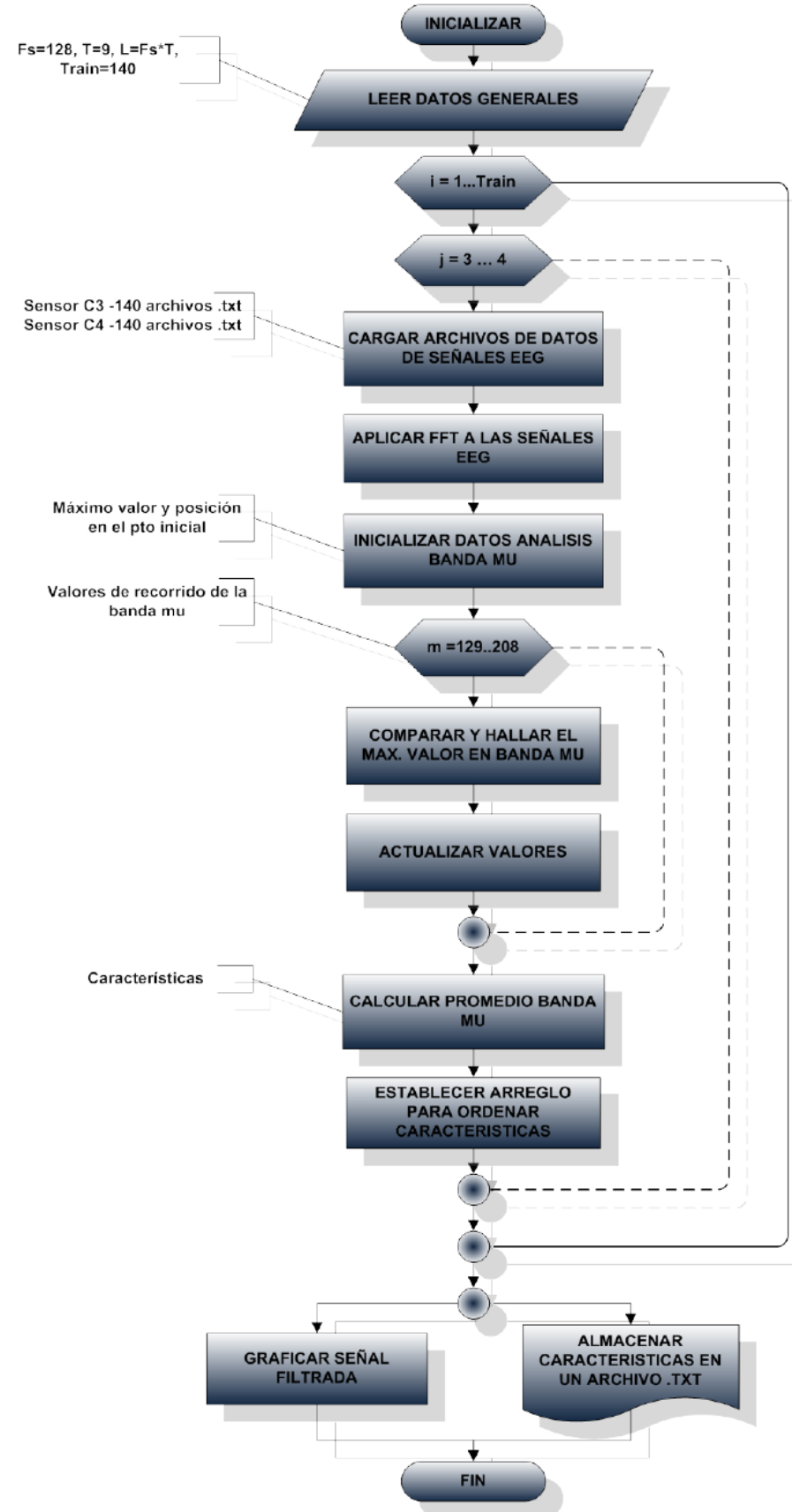

Figura 5: Diagrama de flujo del algoritmo implementado para la extracción de características por el método de la Transformada de Fourier.

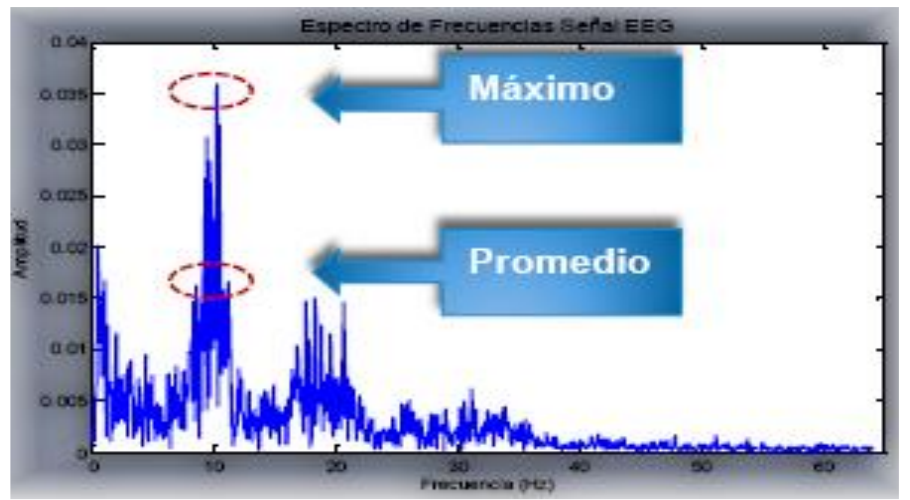

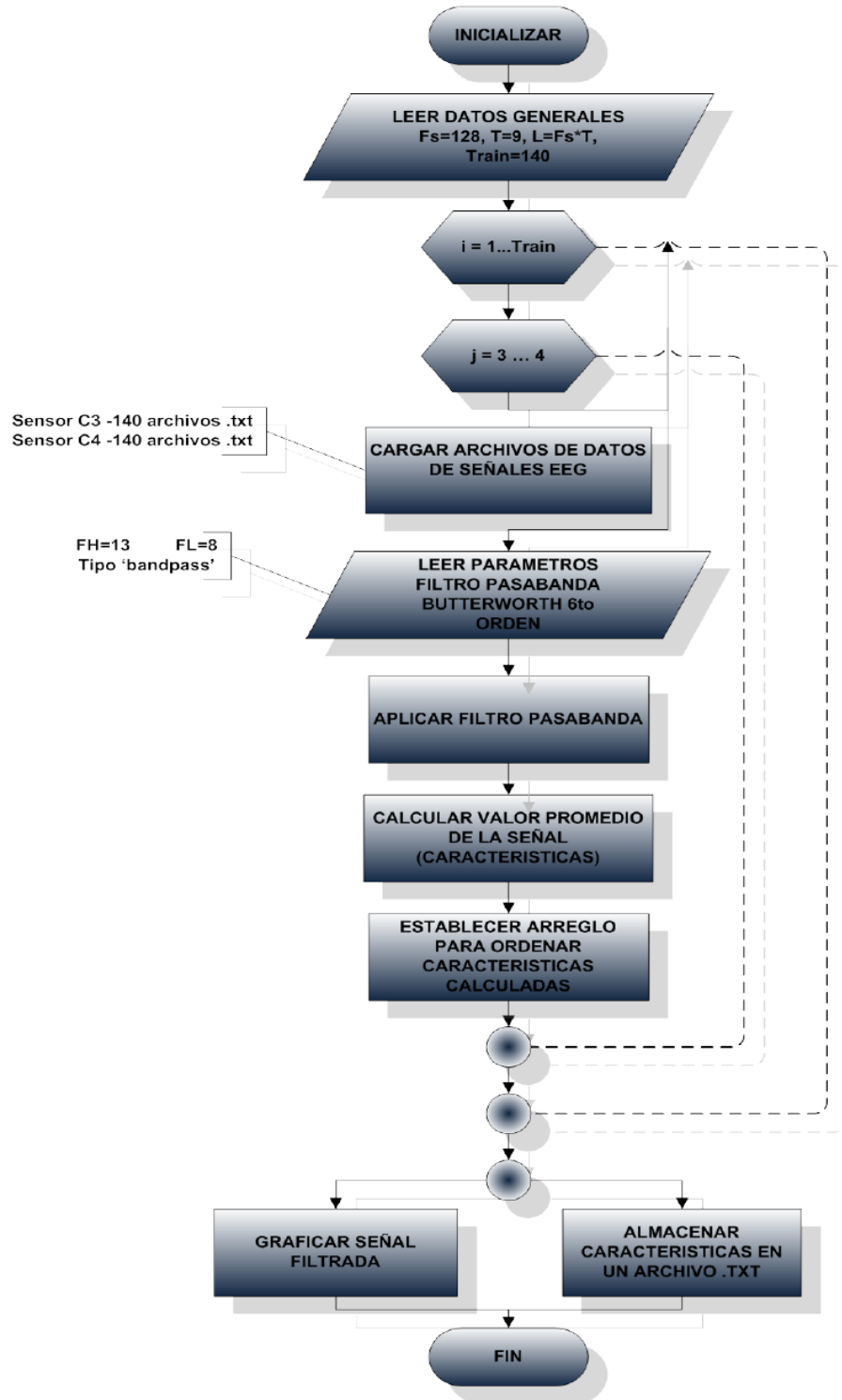

Figure 7: Diagrama de flujo del algoritmo implementado para la extracción de características por el método de Dominio del Tiempo.

Extracción de características para toda la señal en el dominio del tiempo

La serie temporal se define por una secuencia de valores a lo largo del tiempo, el análisis que se hizo para obtener las características de las señales EEG fue mediante la aplicación de un filtro pasa banda Butterworth de sexto orden en la banda de 8 a $13 \mathrm{~Hz}$.

Luego se extraen las características que serán los valores promedio de la Energía de la señal. Se calcularon valores promedio de todas las pruebas realizadas para cada uno de los electrodos, por lo que se obtuvieron 2 características en total para cada prueba. 
Extracción de características en cada instante de tiempo mediante potencia de la señal en las bandas Mu y Beta

En este caso se calculará la Energía Espectral en la banda Mu $(8-13 \mathrm{~Hz})$ y Beta $(16-24 \mathrm{~Hz})$ para cada uno de los electrodos (posiciones C3 y C4) por lo que se tendrá en total un conjunto de 4 características. Las señales EEG fueron segmentadas en bloques de 1s de duración como se muestra en la figura 8. En el análisis en cada instante de tiempo es necesario extraer características en cada instante de tiempo, para lo cual tomamos la ventana de 1s de duración, esta ventana irá desplazándose en cada instante de tiempo; en cada ventana el trabajo que se realiza es el de filtrar la señal primero con un filtro pasa banda Butterworth de sexto orden con una banda de paso para el primero entre $8-13 \mathrm{~Hz}$ (Banda $\mathrm{Mu}$ ) y el segundo para el ritmo Beta con una banda de paso entre $16-24 \mathrm{~Hz}$. Luego los parámetros PST son calculados mediante la fórmula:

$$
P S T=\frac{1}{N} \sum_{n \rightarrow 0}^{N} Y_{t}^{2}
$$

Dónde: $P S T=$ Energía espectral de la señal.

$N=$ Numero de muestras por ventana

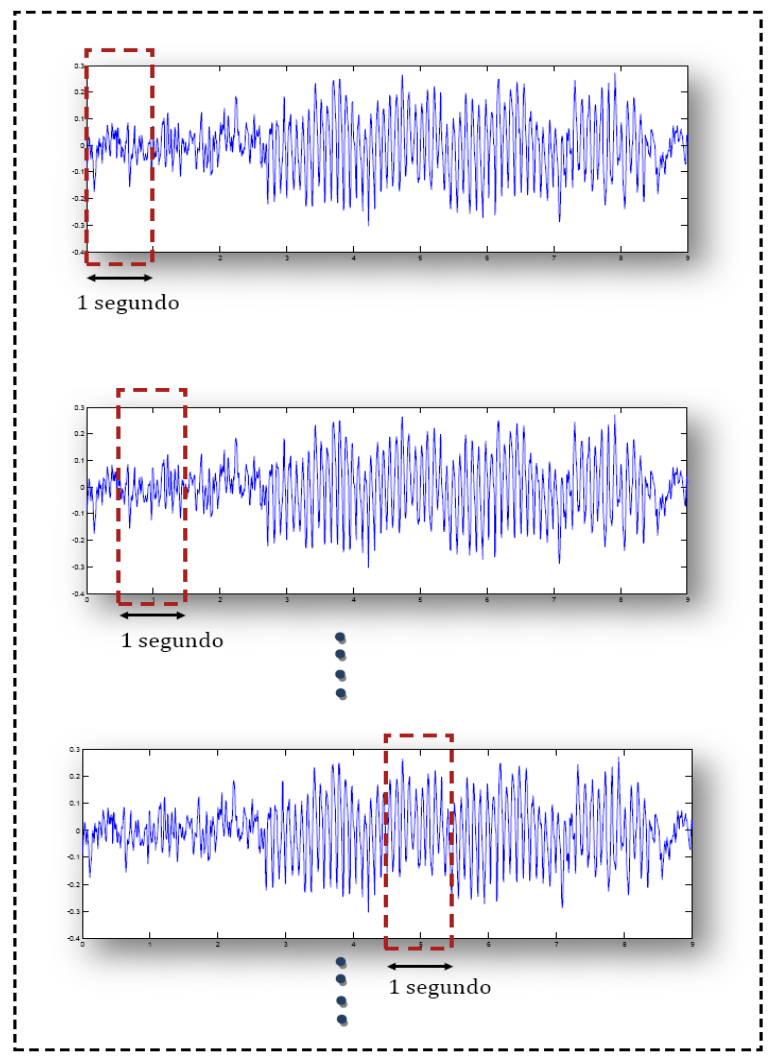

Figura 8: Ventaneado de la señal para obtener la Energía Espectral en las Banda Mu y Beta (total de 2049 ventanas).

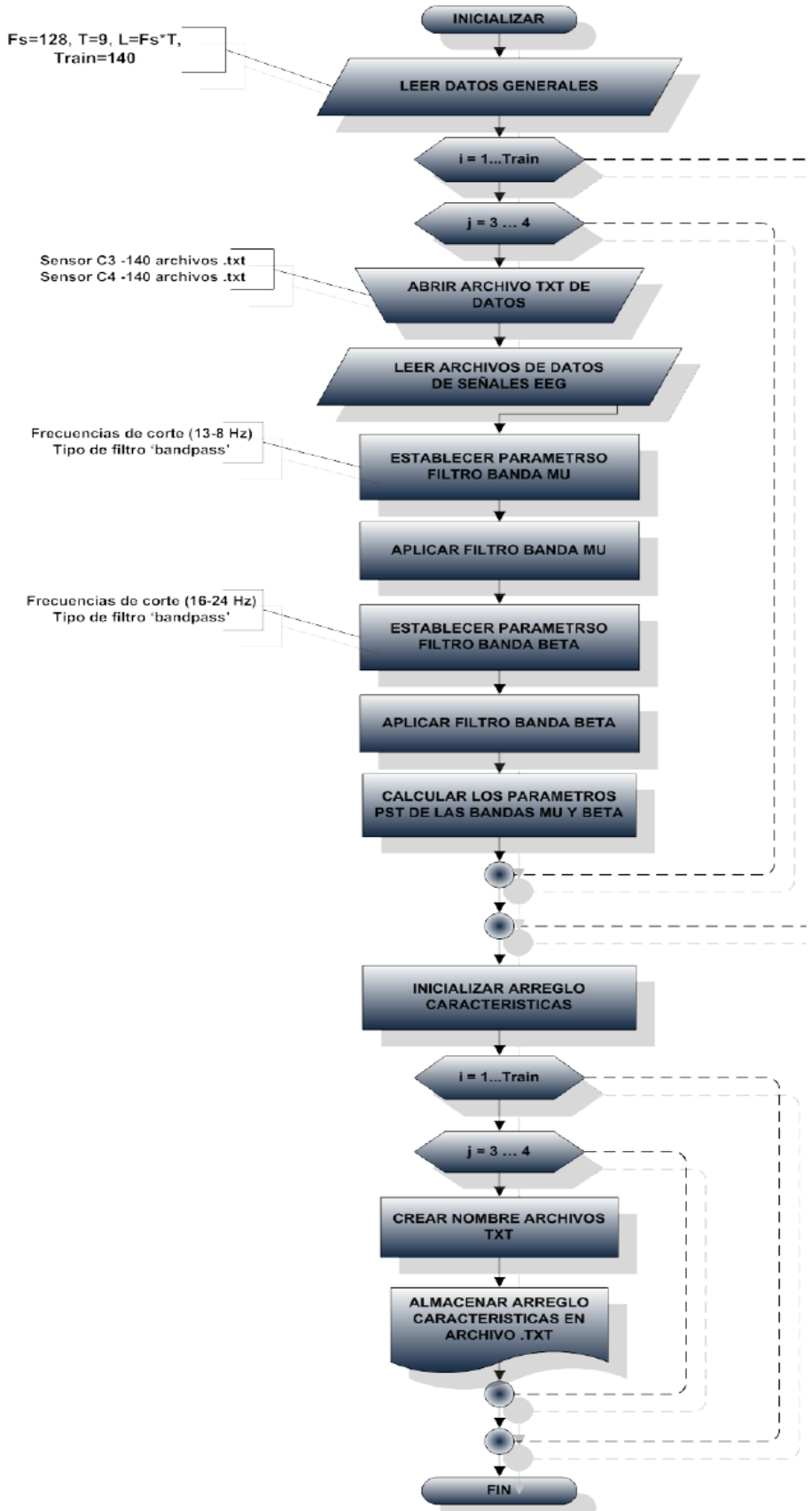

Figura 9: Diagrama de flujo del algoritmo implementado para la extracción de características por el método de Energía Espectral de la Señal.

Extracción de características en cada instante de tiempo mediante parámetros adaptativos autoregresivos

Tal como se presenta en [1], Para representar las características anteriormente descritas en cifras que nos permitan implementar un algoritmo de clasificación, pues utilizamos los parámetros adaptativos autoregresivos que nos permite representar la respuesta en frecuencia. 


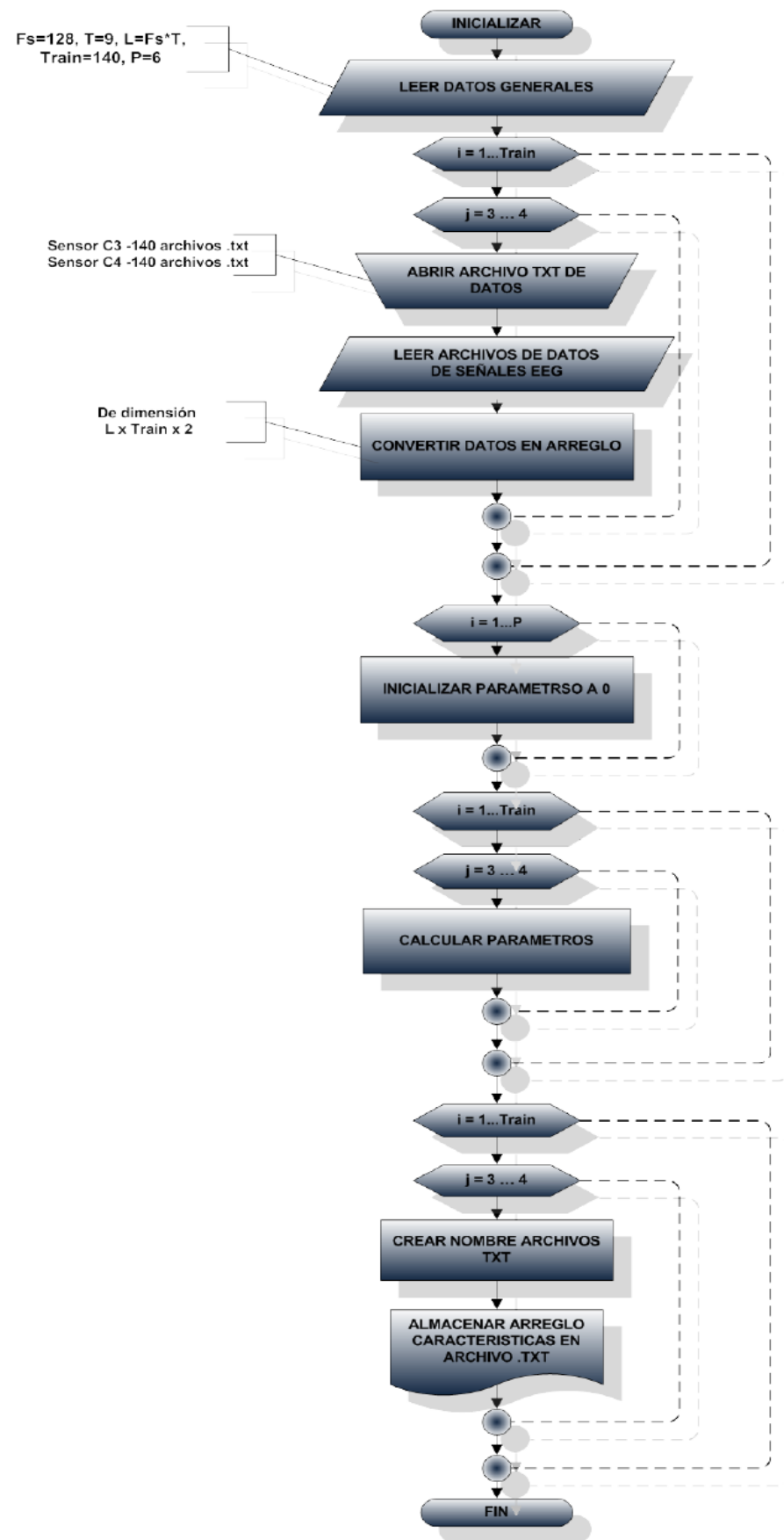

Figura 10: Diagrama de flujo del algoritmo implementado para la extracción de características por el método de Parámetros Adaptativos Autorregresivos.

Un modelo AAR de orden $p$ es escrita de la siguiente manera:

$$
\begin{aligned}
y(t) & =a_{1}(t) * y(t-1)+\ldots+a_{p}(t) * y(t-p)+x(t) \\
& =a(t)^{T} * Y(t-1)+x(t)
\end{aligned}
$$

La predicción de del error se calcula de la siguiente manera:

$$
e(t)=y(t)-\hat{a}(t-1)^{T} * Y(t-1)
$$

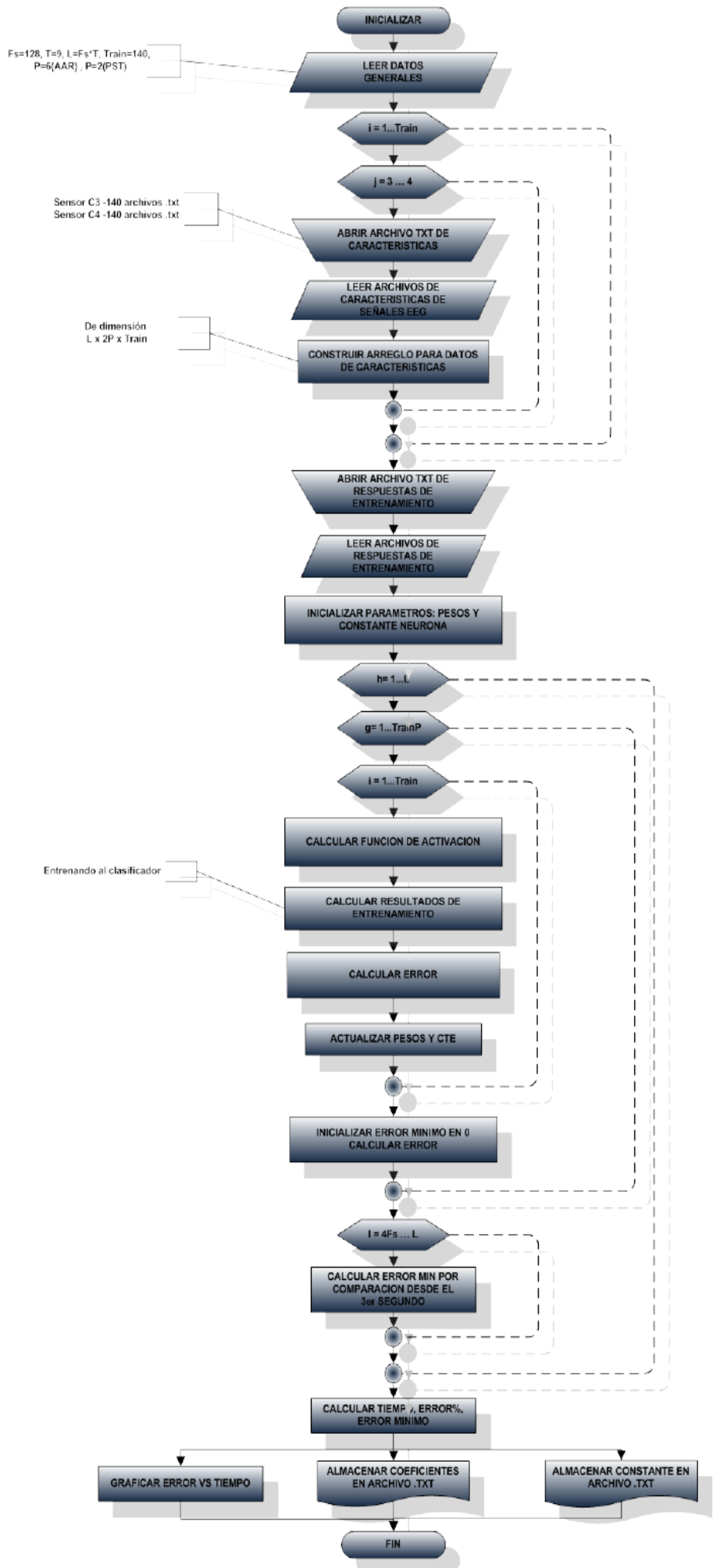

Figura 11: Diagrama de flujo del algoritmo de entrenamiento del clasificador Red Neuronal Artificial.

Para el cálculo de los parámetros existen bastantes métodos, en esta oportunidad utilizamos el método de Last-Mean-Squares (LMS) cuya fórmula está dada por:

$$
\hat{a}(t)=\hat{a}(t-1)+(U C / M S Y) * e(t) * Y(t-1)
$$


$U C \rightarrow$ Coeficient e de Actualizacion $=0.0055$

$M S Y \rightarrow$ Varianzadela Señal $=\frac{1}{N} \sum_{t=1}^{N} Y_{t}^{2}$

En el presenta trabajo se trabajó con 6 parámetros AAR por cada electrodo, es decir u conjunto de 12 características.

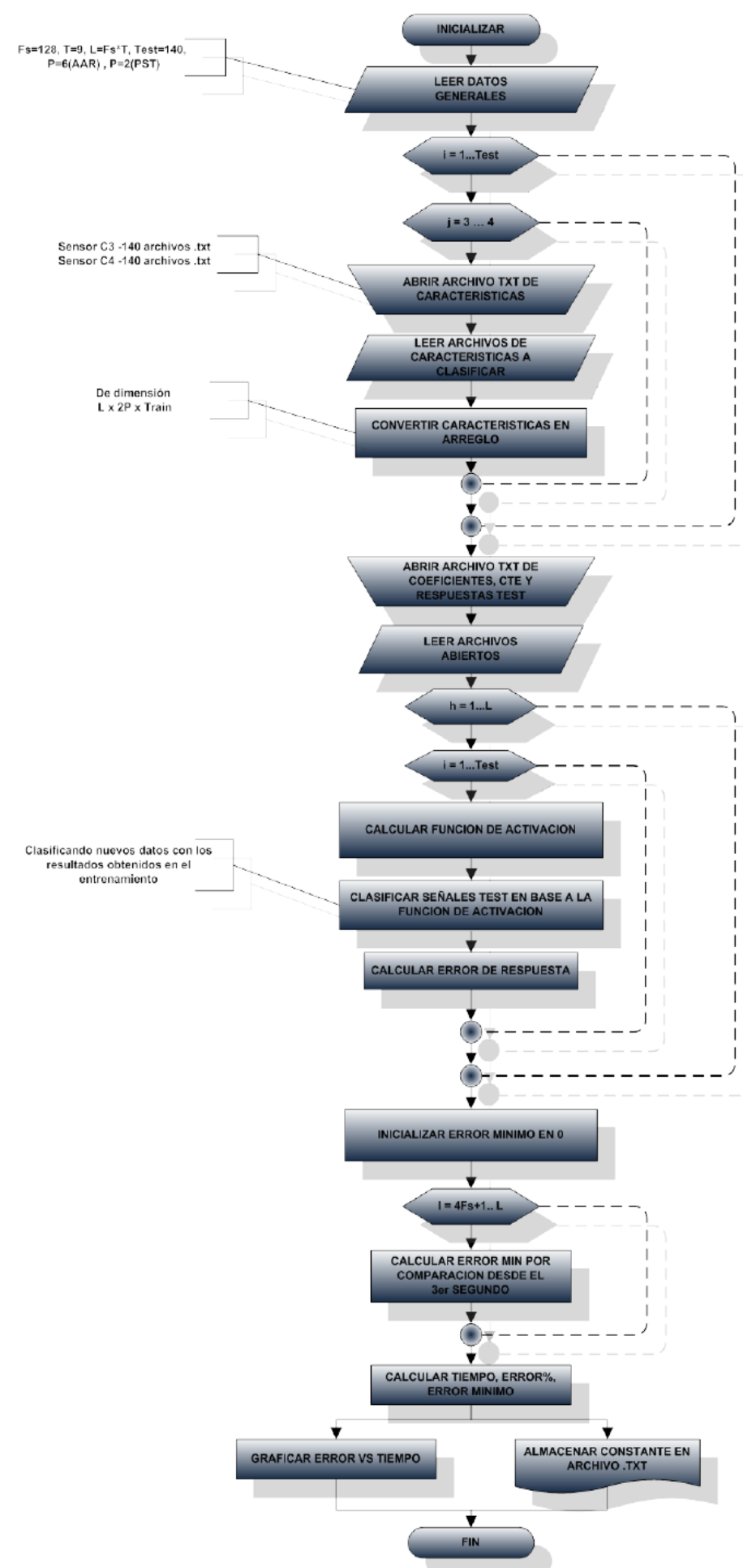

Figura 12: Diagrama de flujo del algoritmo de clasificación por el método de SVM para las características extraídas.

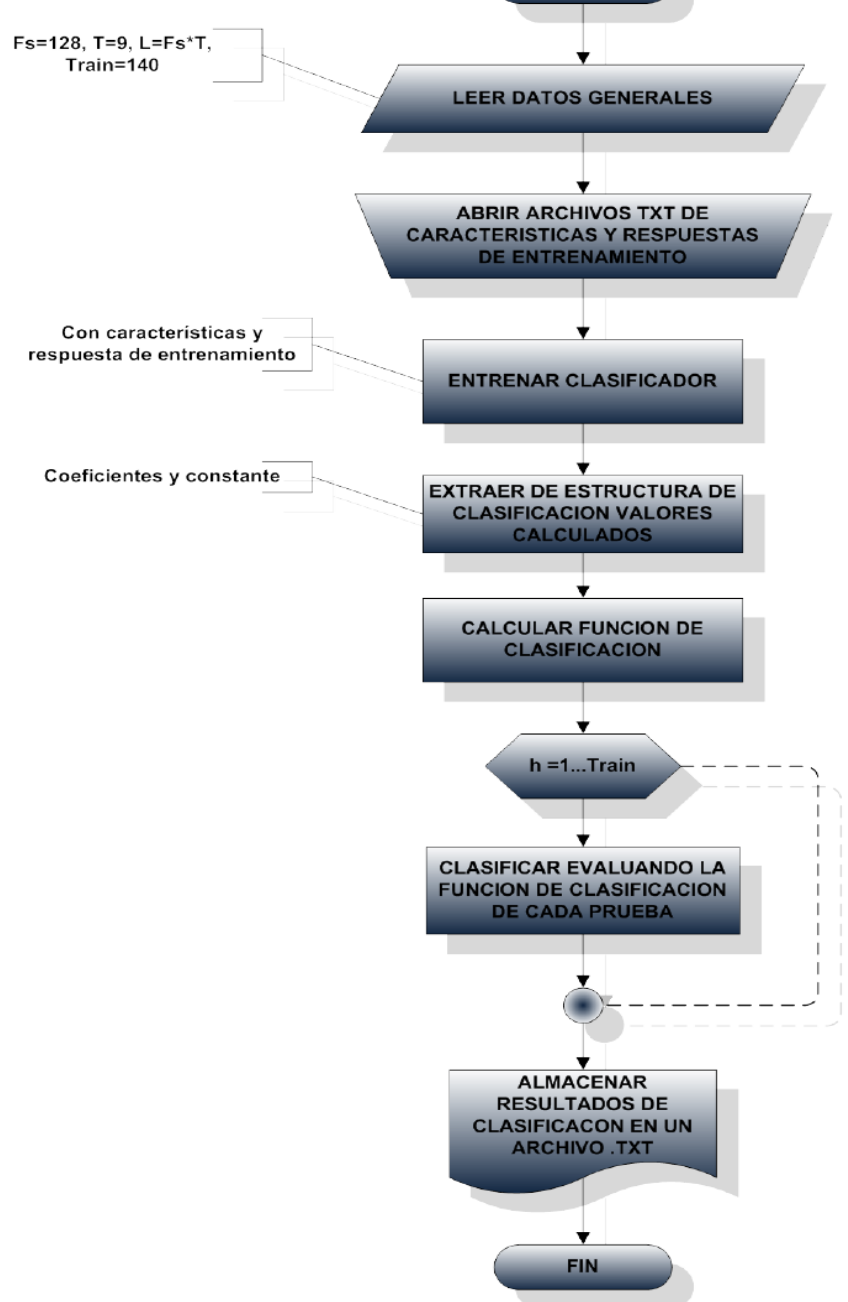

Figura 13: Diagrama de flujo del algoritmo de clasificación por el método de LDA para las características extraídas.

\section{Métodos de Clasificación}

Los métodos utilizados para el propósito de este trabajo en esta etapa son:

- Red Neuronal Artificial

- Máquina de Soporte Vectorial (Support Vector Machine)

- Análisis Discriminante Lineal (Linear Discriminant Analysis)

\section{Resultados}

La evaluación de los diferentes métodos se ha realizado mediante validación cruzada (Cross Validation), porcentaje de error (Error Rate) y cantidad de información mutual (Mutual Information), métodos que son utilizados en [1]; así mismo se han utilizado dos juegos de datos.

La base de datos (Data Set III) de la Universidad de Graz ha sido facilitada por el Departamento de 
Informática Médica durante el evento $\mathrm{BCl}$ Competition 2003. La base de datos contiene 140 pruebas y sus etiquetas, siendo 70 relativas a la imaginación del movimiento de la mano derecha y 70 de la imaginación del movimiento de la mano izquierda. Cada prueba contiene 9s de grabación, a una tasa de muestreo de $128 \mathrm{~Hz}$, lo que genera 1152 muestras/canal/prueba. Los datos se obtienen de manera bipolar alrededor de los puntos C3, Cz y C4, de acuerdo con el sistema internacional 10-20.

La base de datos de la Universidad de Metz, que contiene 60 pruebas y sus etiquetas, siendo 29 relativas a la imaginación del movimiento de la mano derecha y 31 de la imaginación del movimiento de la mano izquierda. La prueba se inicia en reposo y a los 3 segundos el sistema escoge un valor de manera aleatoria para mandar una señal, que será una flecha ya sea apuntando a la derecha o apuntando a la izquierda, por lo que la persona tendrá los 6 segundos restantes para imaginar el movimiento especificado anteriormente.

Al combinar los diferentes métodos de extracción de características y los diferentes métodos de clasificación, finalmente tenemos 12 combinaciones:

- Transformada Rápida de Fourier - Red Neuronal (FFT - RN) Transformada Rápida de Fourier - Máquina de Soporte Vectorial (FFT - SVM).

- Transformada Rápida de Fourier - Análisis Discriminante Lineal (FFT - LDA).

- Dominio del Tiempo - Red Neuronal (DT $\mathrm{RN})$.

- Dominio del Tiempo - Máquina de Soporte Vectorial (DT - SVM).

- Dominio del Tiempo - Análisis Discriminante Lineal (DT - LDA).

- Energía Espectral de la Señal - Red Neuronal (PST - RN).

- Energía Espectral de la Señal - Máquina de Soporte Vectorial (PST - SVM).

- Energía Espectral de la Señal - Análisis Discriminante Lineal (PST - LDA).

- Parámetros Adaptativos Autorregresivos Red Neuronal (AAR - RN).

- Parámetros Adaptativos Autorregresivos Máquina de Soporte Vectorial (AAR - SVM).

- Parámetros Adaptativos Autorregresivos Análisis Discriminante Lineal (AAR - LDA).

Los resultados son los siguientes:

DataSet de la Universidad de Graz

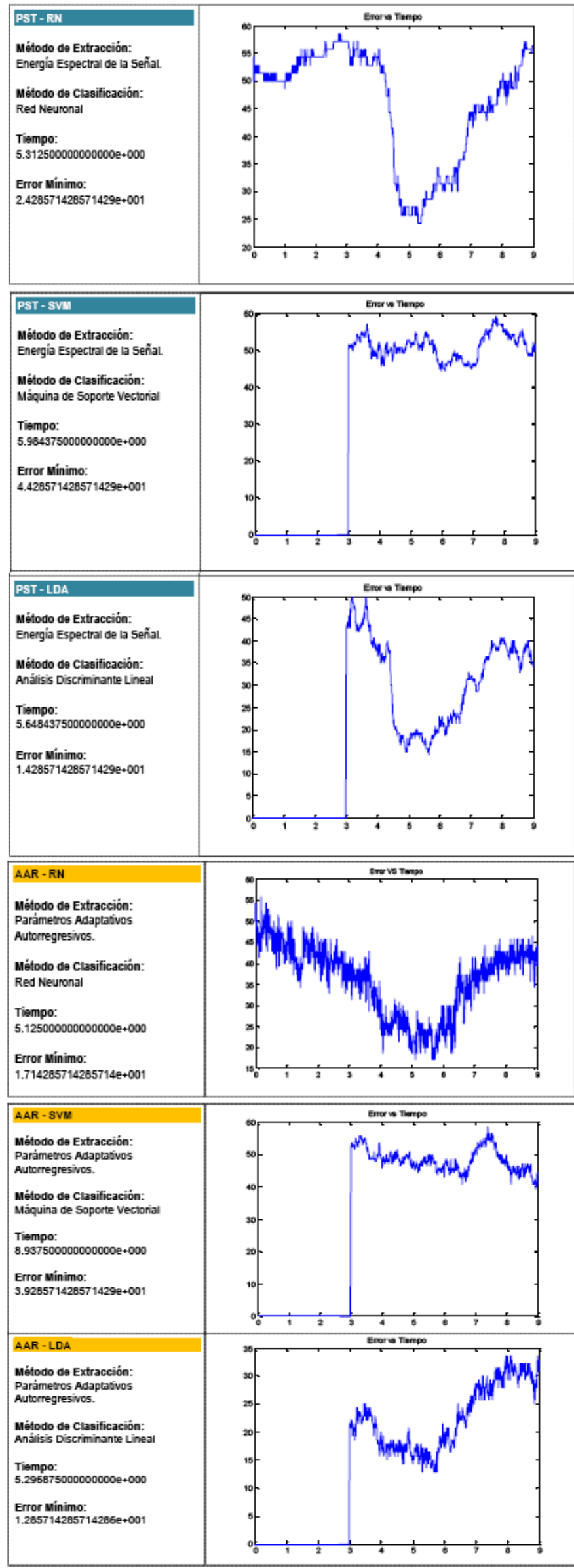


DataSet de la Universidad de Metz

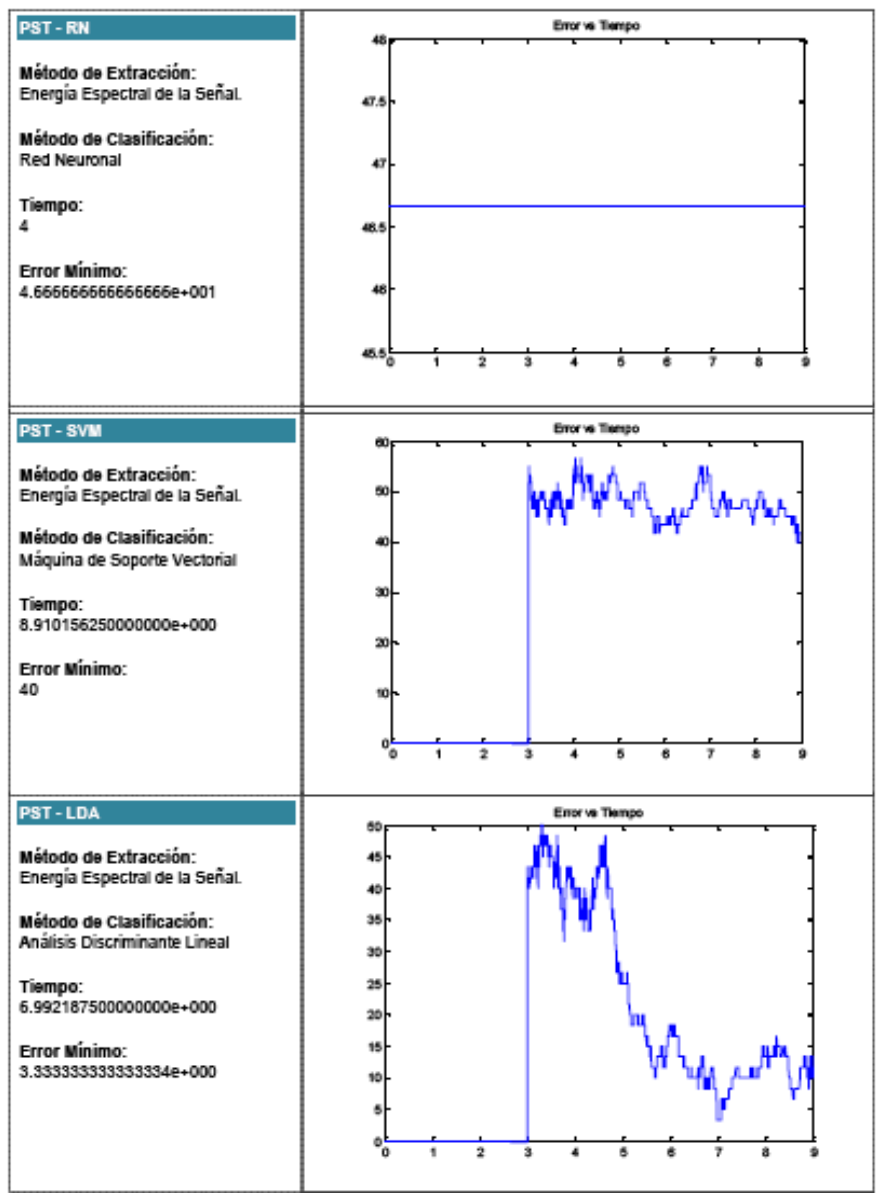

\begin{tabular}{|l|l|}
\hline AAR - RN \\
Método de Extracción: \\
Parámervos Adaptativos \\
Autorregresinos. \\
Método de Clasificación: \\
Red Neuronal \\
Tiempo: \\
7.785156250000000e+000 \\
Error Mínimo: \\
6.666666666666667e+000
\end{tabular}

Resumen de Resultados:

\begin{tabular}{|c|c|c|c|c|c|}
\hline \multicolumn{2}{|c|}{ Cross Validation } & \multicolumn{2}{|c|}{ Error Rate } & \multicolumn{2}{|c|}{ Mutual Information } \\
\hline Métodos & $\%$ Acierto & Métodos & $\%$ Error & Métodos & MI Max \\
\hline AAR - LDA & 88.69 & PST - LDA & 8.81 & PST - LDA & 0.60 \\
\hline PST - LDA & 87.98 & AAR - LDA & 9.77 & AAR - LDA & 0.58 \\
\hline DT - SVM & 81.43 & $A A R-R N$ & 11.91 & AAR - RN & 0.58 \\
\hline FFT - SVM & 80.84 & AAR - SVM & 35.48 & AAR - SVM & 0.20 \\
\hline FFT - LDA & 80.84 & PST - RN & 35.48 & PST - RN & 0.30 \\
\hline$D T$ - LDA & 80.60 & PST - SVM & 42.14 & PST - SVM & 0.34 \\
\hline AAR - RN & 79.77 & DT - SVM & -- & DT - SVM & -. \\
\hline AAR - SVM & 76.43 & FFT - SVM & -- & FFT - SVM & -- \\
\hline PST - SVM & 70.72 & FFT - LDA & -- & FFT - LDA & -- \\
\hline PST - RN & 63.82 & DT - LDA & -- & $D T$ - LDA & -- \\
\hline FFT - RN & 49.17 & FFT - RN & -- & FFT - RN & -- \\
\hline DT -RN & 49.17 & DT -RN & -- & DT -RN & -- \\
\hline
\end{tabular}

\section{Conclusiones}

Sin lugar a duda, los sistemas $\mathrm{BCI}$ resultan de enorme aplicación a la hora de establecer un canal de comunicación para personas con importante discapacidad física, sin embargo aún son muchos los avances que se deben llevar a cabo para asegurar dicho fin.

Los procedimientos de extracción de parámetros, cuando estos son meticulosamente seleccionados, ofrecen mejores resultados y alteran directamente la eficiencia del sistema, por lo cual hacen de esta fase una etapa primordial para el éxito del de un $\mathrm{BCl}$.

De acuerdo al manejo de la señal y la dificultad de elaboración de algoritmos, el análisis de la señal completa es el más sencillo, pero no el más efectivo. El análisis por ventanas brinda mejores resultados ya que se toma la señal por partes y se analiza en cada instante de tiempo de tal forma que se pueda realizar una interacción de manera cuasi continua.

Evaluando el procesamiento hecho con el método de Error Rate se observa que el mínimo error obtenido resulta de la combinación de los parámetros Autorrgresivos con el clasificador LDA en el caso de Graz con un valor igual a 12.86 al igual que los datos de Metz con un valor de 6.66, de igual manera el Cross-Validation nos muestra que el menor porcentaje de error en ambos Dataset también lo da la combinación ya mencionada con valores de 14.29 y 8.33 para los Dataset de Graz y Metz respectivamente.

Mediante evaluación con el Mutual Information, el Dataset de Metz tiene la mejor combinación para el procesamiento mediante la Energía Espectral y el Aálisis Discriminante Lineal y en el caso del Dataset 
de Graz la combinación AAR y LDA es la más efectiva con valor máximo de MI igual a 0.53.

\section{Referencias}

[1] A. Roman Gonzalez, "System of Communication and Control based on the Thought", IEEE International Conference on Human System Interaction - I'10, Poland, May, (2010) 275-280.

[2] A. Roman Gonzalez, "Communication Technologies Based on Brain Activity", World Congress in Computer Science, Computer Engineering and Applied Computing WORLDCOMP 2010, Las Vegas, Nevada, USA, July (2010) 864-869.

[3] U. Hoffmann, J. Vesin, T. Ebrahimi, "Recent Advances in Brain-Computer Interfaces", Ecole Polytechnique Federale de Laussanne (EPFL), Switzerland (2007).

[4] J. R. Wolpaw, N. Birbaumer, D. J. McFarland, G. Pfurtscheller, T. M. Vaughan, "BrainComputer Interfaces for Communication and Control", Clinical Neurophysiology, 113 (2002) 767-791.

[5] C. Lecocq, F. Cabestaing, "Les Interfaces Cerveau-Machine pour la Palliation du Handicap Motor Severe", LAGIS - Laboratoire d'Automatique, Génie Informatique \& Signal, Université des Sciences et Technologie de Lille, (2008).

[6] J. C. Lee, D. S. Tan, "Using a Low-Cost Electroencephalograph for Task Classification in $\mathrm{HCl}$ Research", Montreux Switzerland, (2006).

[7] S. Kuo-Kai, L. Po-Lei, L. Ming-Huan, L. MingHong, L. Ren-Jie, Ch. Yun-Jen, "Development of a Low-Cost FPGA-Based SSVEP BCl Multimedia Control System", IEEE Transaction on Biomedical Circuits and Systems, 4 (2010) 125-132.

[8] M. Kirby, "Some Mathematical Ideas for Attacking the Brain Computer Interface
Problem", Departement of Mathematics, Colorado State University.

[9] F. Alarid-Escudero, T. Solis-Escalante, E. Melgar, R. Valdes-Cristerna, O. YañezSuarez, "Registro de Señales de EEG para Aplicaciones de Interfaz Cerebro Computadora (ICC) Basado en Potenciales Evocados Visuales de Estado Estacionario (PEVEE)", Universidad Autónoma Metropolitana, México.

[10] G. Pfurtscheller, F. H. Lopes da Silva, "Eventrelated EEG/MEG Synchronization and Desynchronization: Basic Principles", Clinical Neurophysiology, 110 (1999) 1842-1857.

[11] B. Kamousi, Z. Liu, B. He, "Classification of Motor Imagery Tasks for Brain-Computer Interface Applications by Means of Two Equivalent Dipoles Analysis", IEEE Transaction on Neural Systems and Rehabilitation Engineering, 13 (2005) 166-171.

[12] A. Kachenoura, L. Albera, L. Senhadji, P. Comon, "ICA: A Potential Tool for $\mathrm{BCl}$ System", IEEE Signal Processing Magazine, 25 (2008) 57-88.

[13] F. Faradji, R. K. Ward, G. E. Birch, "A BrainComputer Interface Based on Mental Task with Zero False Activation Rate", IEEE EMBS Conference on Neural Engineering, (2009).

[14] K. Tavakolian, F. Vasefi, K. Naziripour, S. Rezaei, "Mental Task Classification for Brain Computer Interface Applications", Canadian Student Conference on Biomedical Computing.

E-mail: avid.roman-gonzalez@ieee.org 